Musées, Patrimoine et Culture scientifiques et techniques

$186 \mid 2019$

novembre-décembre 2019

\title{
Pour un débat ouvert et partagé
}

Fems

\section{OpenEdition}

Journals

Édition électronique

URL : http://journals.openedition.org/ocim/3365

DOI : 10.4000/ocim.3365

ISSN : 2108-646X

Éditeur

OCIM

Édition imprimée

Date de publication : 1 novembre 2019

Pagination : 15-17

ISSN : 0994-1908

Référence électronique

Fems, « Pour un débat ouvert et partagé », La Lettre de I'OCIM [En ligne], 186 | 2019, mis en ligne le 01 novembre 2020, consulté le 25 janvier 2021. URL : http://journals.openedition.org/ocim/3365; DOI : https://doi.org/10.4000/ocim.3365

Ce document a été généré automatiquement le 25 janvier 2021.

Tous droits réservés 


\section{Pour un débat ouvert et partagé}

\section{Fems}

1 La Fédération des écomusées et des musées de société (Fems) n'est pas restée insensible et immobile face à la polémique ouverte durant l'été - avec le post d'Icom le 25 juillet dernier sur son site internet et les réseaux sociaux - d'une proposition de nouvelle définition du musée, qui devait être soumise au vote des adhérents durant l'assemblée générale extraordinaire de la conférence générale d'Icom 2019 à Kyoto.

2 Malgré une période peu propice à l'échange au sein de notre fédération et l'absence de représentation à la conférence générale à Kyoto début septembre, le conseil d'administration a rédigé une contribution, envoyée à la présidente d'Icom, Suay Aksoy, ainsi qu'à la présidente d'Icom France, Juliette Raoul-Duval, dont la Fems est membre de droit.

3 Pour produire cette contribution, nous avons échangé entre nous, sous forme d'un fil de discussion, et agrégé ces premières réflexions, qui doivent être prises pour ce qu'elles sont : « une réaction à chaud », initiée par Céline Chanas, présidente de la Fems, enrichie des remarques et contributions d'Hugues de Varine, «père » des écomusées et ancien directeur de l'Icom de 1965 à 1974, d'Alexandre Delarge, ancien directeur de l'écomusée de Fresnes et ancien président de la Fems et de Joëlle Le Marec, professeur en sciences de l'information et de la communication, membre associée à la Fems. Cette contribution a été approuvée par l'ensemble du conseil d'administration de la Fems et diffusée à nos adhérents début septembre.

4 Alors que notre fédération s'emploie depuis sa création en 1989 à apporter des éléments de réflexion sur l'évolution de la notion de musée, à re-questionner son rôle dans la société, il nous paraît primordial de prendre part à ce débat en tant que membre de droit d'Icom France, et de faire connaitre le point de vue des écomusées et musées de société qui forment notre réseau.

5 Et ce d'autant plus que notre année 2019 est placée sous le signe de l'héritage de Georges Henri Rivière, un des fondateurs d'Icom et son premier directeur. Après la journée d'étude qui lui a été consacrée à Marseille le 18 janvier dernier, la Fems appelle ses membres à venir contribuer aux orientations de notre nouveau projet fédératif dans un séminaire programmé les 4 et 5 décembre 2019. 
6 En termes de méthode, nous rejoignons la position d'Icom France et regrettons de n'avoir pas pu - su - mieux mobiliser nos adhérents et leurs institutions dans un délai aussi court.

7 Les réflexions qui suivent ne sont donc que l'esquisse de réactions à chaud qui nécessiteront d'être partagées, débattues, synthétisées dans nos prochaines rencontres: l'opportunité se présentera lors du séminaire fédératif de décembre à l'écomusée de Fresnes, puis lors de l'assemblée générale 2019, qui se tiendra dans le cadre de nos prochaines rencontres professionnelles, fin mars 2020 à Bordeaux. À l'écomusée des monts d'Arrée, au sein du Parc naturel d'Armorique, s'est également tenue le 9 octobre une journée de réflexion ouverte à tous, pour proposer une relecture de la notion d'écomusée, au prisme des enjeux actuels.

8 Avec cette nouvelle définition du musée proposée par l'Icom, nous songeons rétrospectivement à plusieurs événements majeurs qui ont déjà invité à un repositionnement du musée et ont alimenté les réflexions sur son rôle social : la 9e conférence de l'Icom à Grenoble en 1971 avec pour thème "Le musée au service des hommes, aujourd'hui et demain " et la table-ronde de Santiago du Chili, organisée sous l'impulsion d'Hugues de Varine en 1972 sous l'égide de l'Unesco, tournant dans la muséologie avec le développement des musées communautaires. La définition des écomusées et musées de société de la Fems adoptée en 2011 constitue aussi une évolution et un élargissement de la définition évolutive de Georges Henri Rivière (GHR).

9 La Fédération des écomusées et des musées de société se réjouit donc que la définition du musée soit re-questionnée, mais elle appelle à un travail de plus long terme, inclusif et démocratique.

\section{Une définition trop complexe}

10 La définition proposée comporte des orientations intéressantes, dans lesquelles écomusées et musées de société se retrouvent largement, mais sa formulation nous semble inutilement complexe, verbeuse voire jargonnante.

11 La définition précédente, certes imparfaite, avait le mérite d'être intelligible et compréhensible par le plus grand nombre. Nous prônons donc un travail de synthèse pour parvenir à une définition compréhensible dès la première lecture, y compris pour des non-initiés.

\section{Le musée, entre missions traditionnelles et nouveaux engagements}

Cette définition est étonnante : la position critique défendue, certes très contemporaine dans les sciences sociales, ne nous semble pas être de nature à définir une institution aussi complexe et plurielle que l'ensemble des musées concernés. Elle nous semble confondre définition et éléments d'une perspective théorique et critique, qui trouveraient mieux leur place dans d'autres types de documents, comme le code de déontologie. 
dernier à Montréal, la notion d'inclusion y est interrogée du point de vue de la politique des publics, de leur projet de diffusion culturelle et scientifique, de leur politique managériale, mais aussi de leur modèle économique. L'ouvrage Le musée participatif: l'ambition des écomusées issu des réflexions de la Fems va jusqu'à prôner l'inclusion des habitants dans la conception des établissements et de leurs actions. Depuis une vingtaine d'années, de nombreuses initiatives visant à inclure et laisser la place à différents publics dans les musées, sont à l'œuvre dans les musées de notre réseau. Quant au récit que proposent nos musées, ils s'inscrivent justement dans le principe d'expertises partagées et d'ouverture vers les communautés, les habitants. Cette polyphonie est la condition même d'un processus de patrimonialisation ouvert, co-construit.

\section{Définition de l'écomusée}

« L'écomusée est une institution culturelle assurant, d'une manière permanente, sur un territoire donné, avec la participation de la population, les fonctions de recherche, conservation, présentation, mise en valeur d'un ensemble de biens naturels et culturels, représentatifs d'un milieu et des modes de vie qui s'y succèdent ». Charte des écomusées, 2011

A contrario, on voit difficilement les grands musées, enrôlés dans des politiques de développement touristique, pouvoir sérieusement incarner cette définition, sauf en créant des services un peu périphériques, destinés à créer de la communication plus qu'à structurer leur fonctionnement tout entier autour des éléments de la nouvelle définition. Or, même si ces musées ne sont pas ceux dont nous nous sentons les plus proches, ils ont leur place dans la diversité des institutions et ne doivent pas en être exclus.

17 La définition du musée, elle aussi, doit être inclusive et nous rassembler plutôt que nous diviser.

On voit également assez mal comment les missions historiques de conservation et de transmission - "le musée acquiert, conserve, expose, transmet"-, extrêmement structurantes et qui ont forgé des équilibres qu'il faut sans cesse entretenir, pourraient soudain être mises au second plan au nom d'une définition qui nierait ce que sont et font objectivement les musées existants.

19 Cette définition risquerait de faire rapidement apparaître comme vides et décevantes toutes les belles notions affichées, et donc de détruire leur portée réelle. 


\section{Des lieux de projets plutôt que des lieux de démocratisation?}

De même, nous ne voyons pas pourquoi les musées seraient des lieux de démocratisation, ce qui signifie qu'ils seraient là pour apporter ce qui n'est pas encore existant.

Il nous semble que la définition de la Fems, qui affirme que le musée est un lieu de projet, est plus intéressante que celle-ci qui dit que c'est un «lieu qui reconnaît et aborde les conflits et les défis".

Pourquoi, en effet, mettre en avant le fait que le musée est un lieu "reconnaissant et abordant les conflits et les défis du présent ", car on pourrait tout aussi bien dire qu'il exprime la fraternité et l'entretien quotidien de ce qui fait la vie des populations et des sociétés, en se référant par exemple à l'éthique du care et non uniquement aux défis, à la conflictualité...

Il nous apparaît que plutôt que de conserver « des mémoires diverses ", ce qui postule un choix et donc une sélection, il faudrait conserver "toutes les mémoires ", même si c'est un peu utopique, mais la définition évolutive de GHR n'était-elle pas utopique et, de ce fait, heuristique?

De même les musées, dans leur très grande diversité, doivent travailler pour toutes les communautés et non « diverses communautés ».

Il faut que l'égalité des droits et accès soit pour tous les citoyens et non pour tous les peuples, ce qui au bout du compte rend un peu abstrait la caractérisation des populations et des publics.

Dans le même esprit, est-ce la compréhension du monde ou celle du territoire à laquelle il faut œuvrer? Le monde nous semble trop vaste et flou. Le musée se construit sur un territoire, qui en fait sa propre singularité.

Par ailleurs, le terme " améliorer » apparaît un peu surplombant car il postule qu'il faut guider les gens et non élaborer avec eux la compréhension des sujets saisis. Les petites communautés territoriales ont aussi des musées participatifs qui n'ont pas la prétention de démocratiser mais d'être au service de la population (et de ce fait dans des démarches de démocratie participative), et qui agissent très concrètement non pas pour « le monde », mais dans des territoires précis. Ils ne sont pas les brouillons ou les signes d'autre chose, ce à quoi risque de les renvoyer la définition.

relisant la définition proposée, on ne peut que souhaiter de parvenir à une nouvelle définition, qui soit à la fois simple et consensuelle. Nous pensons qu'il faut se donner encore du temps et y travailler collégialement. constituent notre contribution actuelle à ce travail auquel la Fems et ses membres sont prêts à participer.

Nous aimerions échanger sur des notions et principes tels que : le musée est un espace ouvert et permanent; le musée associe des acteurs et des agents (quel que soit le nom donné à ces ensembles, qui pourraient être salariés et bénévoles); le musée constitue, conserve et diffuse les patrimoines naturels, culturels, matériels et immatériels; le musée est un processus qui postule que les hommes et les projets peuvent changer en fonction des évolutions du territoire. 


\section{RÉSUMÉS}

Cet article est extrait du dossier «Vers une nouvelle définition du musée ? » dirigé par Ewa Maczek, directrice par intérim de l'Ocim. La définition du musée proposée depuis 2007 par l'Icom fait référence dans la sphère muséale mondiale. Si la volonté de faire évoluer cette définition fait sens, la proposition du Comité pour la Définition du musée, perspectives et potentiels (MDPP) de l'Icom a surpris bon nombre de professionnels et d'institutions, et tout particulièrement en France. La lettre de l'Ocim leur a ouvert ses pages pour qu'ils partagent leurs réactions.

\section{INDEX}

Mots-clés : politique du patrimoine, organisation internationale

\section{AUTEUR}

FEMS

Fédération des écomusées et des musées de société contact@fems.asso.fr 